\title{
Consulta adolescente en servicios de emergencia pediátrica
}

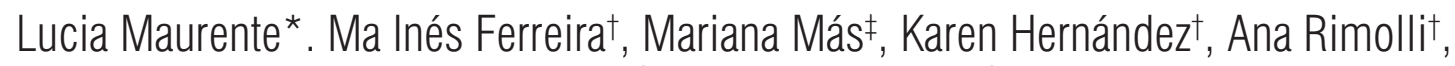
Florencia Castellanos ${ }^{\dagger}$, Javier Prego§, Gustavo Giachetto§, Loreley García

\section{Resumen}

La Organización Mundial de la Salud define como adolescencia la etapa comprendida entre los 10 y 19 años (temprana: 10 a 14 años; tardía: 15 a 19 años). La prevalencia de enfermedades en la adolescencia es baja; sin embargo, los adolescentes utilizan los servicios de emergencia, lo que requiere un abordaje integral y multidisciplinario. No hay datos publicados sobre los motivos de consultas de adolescentes en Uruguay.

Objetivo: conocer la frecuencia y las características de la consulta de adolescentes en los servicios de emergencia del subsector público y privado del sistema nacional integrado de salud.

Metodología: estudio multicéntrico, retrospectivo, de adolescentes de entre 10 y 14 años que consultaron en el Hospital Pediátrico-Centro Hospitalario Pereira Rossell de la Administración de los Servicios de Salud del Estado, subsector público, y entre 10 y 19 años en la Asociación Española Primera de Socorros Mutuos, en el Centro de Asistencia Médica del Oeste de Colonia y en Casa de Galicia. Se incluyeron las consultas registradas entre los días 7 y 13 de los meses de enero, abril, julio y octubre del 2013. Se registraron: edad, sexo, motivo de consulta, comorbilidades, consultas con especialistas, exámenes de laboratorio, diagnóstico y destino al egreso. Se realizó análisis estadístico, considerando estadísticamente significativo $p<0,05$.

Resultados: $n=1.518,9 \%$ del total de consultas. Edad: media 13 años 1 mes, varones 50,5\%; tenía comorbilidades: $26 \%$. Subsector público 35\%; subsector privado, instituciones de asistencia médica colectiva (IAMC) $65 \%$. Distribución de consultas por mes: enero $20 \%$, abril $30 \%$, julio $20 \%$, octubre $30 \%$. Los motivos de consulta más frecuentes fueron lesiones e injurias: $33 \%$; síntomas respiratorios: $14 \%$; otros: $13 \%$; síntomas digestivos: $12,5 \%$; lesiones en piel: $7 \%$. Los problemas psicosociales fueron $7,2 \%$ en el sector público, en tanto que en el privado fue $3,2 \%$. Consulta con especialista: $38 \%$. Laboratorio: $16 \%$. Imágenes: 30\%. Diagnósticos principales al egreso: trauma $33 \%$; patología respiratoria $20 \%$; otros $13 \%$; patología digestiva $10 \%$; patología de piel 6,6\%. Destino final: domicilio 90\%; hospitalización en el subsector público 13\%; en IAMC 8\%. El 0,9\% se retira antes de la asistencia, $0,1 \%$ se institucionaliza.

Conclusiones: si bien la consulta de adolescentes es de baja prevalencia, la misma genera un impacto en los servicios de urgencia por la elevada utilización de recursos. Se observan diferencias entre el sector público y el privado.

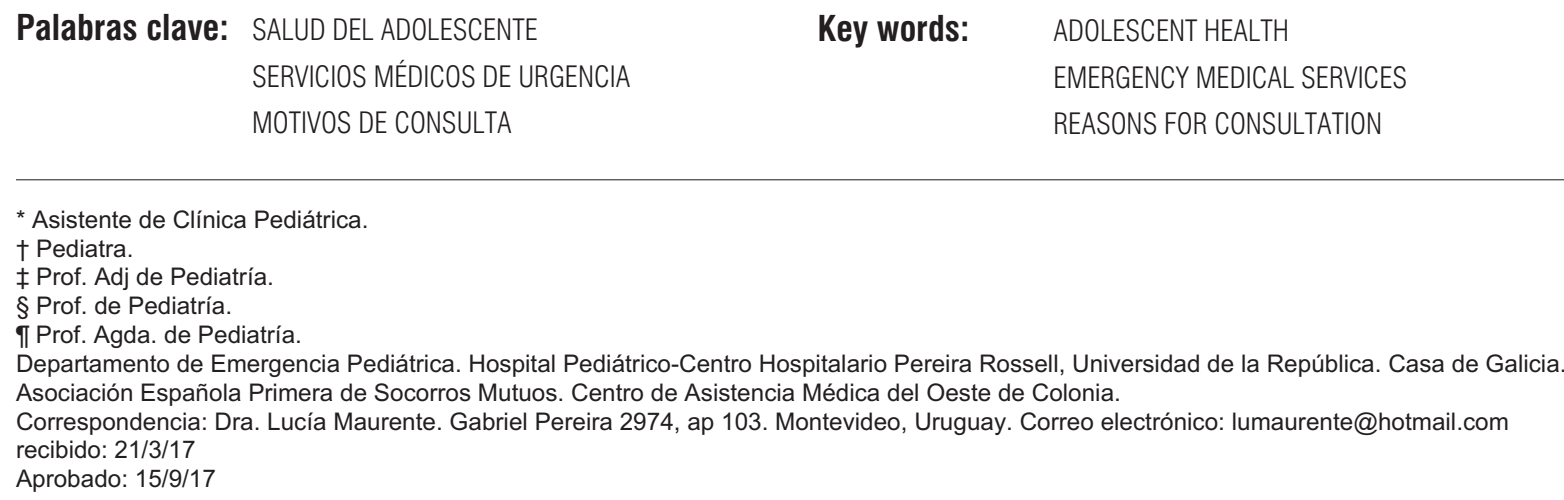




\section{Introducción}

La adolescencia es una de las etapas en la que se producen los mayores cambios en la vida del ser humano. La pubertad provoca modificaciones fisiológicas, emocionales, cognitivas y del comportamiento que impactan en los vínculos familiares y sociales. Si bien la prevalencia de enfermedades en la adolescencia es menor que en otras etapas de la vida, esto no implica que los requerimientos de los servicios de atención de salud sean menores $^{(1-3)}$.

Las características propias de las personas en la edad de la adolescencia promueven la experimentación en situaciones de riesgo que pueden constituirse en lesiones transitorias o daños permanentes. Desde esta perspectiva, los adolescentes constituyen un grupo especialmente vulnerable y frecuentemente expuesto a situaciones que amenazan su salud, independientemente del nivel socioeconómico y cultural al que pertenecen. El perfil epidemiológico de la morbimortalidad de los adolescentes cambió en comparación a décadas anteriores. Lesiones, injurias, intentos de autoeliminación y suicidio, trastornos alimentarios, embarazo no deseado, adicciones y enfermedades de transmisión sexual son los problemas que más impactan en la salud de los adolescentes. Los niños con patologías crónicas alcanzan con mayor frecuencia la adolescencia, presentando nuevos desafíos para el abordaje terapéutico. Estos problemas requieren un abordaje integral, multidisciplinario e intersectorial $^{(1-5)}$.

La Organización Mundial de la Salud (OMS) define la adolescencia como la etapa comprendida entre los 10 y 19 años, dividiéndola en adolescencia temprana entre 10 y 14 años y tardía entre 15 a 19 años respectivamente ${ }^{(6)}$. El 2\% de las personas entre 10 a 20 años es hospitalizado durante la adolescencia ${ }^{(1-4)}$, cifra en continuo ascenso por la creciente tasa de morbimortalidad asociada a injurias y patologías, como abuso de drogas, trastornos de la conducta alimentaria, complicaciones por interrupción del embarazo y enfermedades de transmisión sexual $^{(4,5,7)}$.

Si bien se dispone de la Encuesta Nacional de Salud Adolescente (ENSA), que aporta datos sobre la situación de la salud adolescente en Uruguay, no se dispone de información en los prestadores integrales del Sistema Nacional Integrado de Salud (SNIS). Esta información es relevante para la planificación y organización de los servicios. Así mismo, para conocer la necesidad de capacitación de los recursos humanos en el área.

\section{Objetivo}

Conocer la frecuencia y las características de la consulta de adolescentes en servicios de emergencia de un hospi- tal del subsector público y de tres instituciones de asistencia médica colectiva (IAMC) del SNIS.

\section{Metodología}

Se realizó un estudio descriptivo, multicéntrico, retrospectivo, donde se incluyeron adolescentes de entre $10 \mathrm{y}$ 14 años de edad que consultaron en el Hospital Pediátrico del Centro Hospitalario Pereira Rossell (HP-CHPR), del subsector público, y personas de entre 10 y19 años de edad asistidas en Asociación Española (AE), Cooperativa Asistencial Médica Oeste de Colonia (CAMOC) y Casa de Galicia (CG) de tres IAMC.

Se incluyeron las personas que consultaron entre los días 7 al 13 y que consultaron en los meses de enero, abril, julio y octubre del 2013. Se eligieron cuatro meses del año representativos de cada estación para evitar el sesgo estacional. La segunda semana del mes fue seleccionada para evitar el sesgo de la disminución de consultas por la limitante económica de acceso a los servicios de salud.

Las variables analizadas fueron: edad, sexo, motivo de consulta, comorbilidades (enfermedades que se añaden a la enfermedad inicial), consultas con especialistas, exámenes de laboratorio, diagnóstico al egreso y destino del paciente.

Se incluyeron en la categoría trauma a los siniestros de tránsito, las lesiones durante deportes recreativos, quemaduras, heridas, asfixia por inmersión y caída de altura.

En la patología respiratoria se incluyeron la patología infecciosa y enfermedades crónicas (asma, enfermedad pulmonar crónica, fibrosis quística).

En la patología digestiva se incluyeron patología gastroduodenal, cólicos biliares, trastornos del tránsito por patología médica, infecciones gastrointestinales y enfermedades crónicas.

En la patología de piel se incluyeron la patología infecciosa e inmunoalérgica.

Se definió patología psicosocial como el conjunto de patologías psiquiátricas (trastornos de personalidad, crisis de excitación, intento de autoeliminación), más situaciones sociales tales como maltrato, abuso sexual, situaciones de violencia, violencia de género, domésticas u otras y constatación de lesiones.

Se incluyeron en patología urinaria patología infecciosa y uropatías.

En la patología neurológica se incluyeron patología infecciosa y no infecciosa del sistema nervioso.

En patología quirúrgica se incluyeron patologías quirúrgicas abdominales y genitales.

En consultas relacionadas con salud sexual y reproductiva se incluyeron patología infecciosa, consultas vinculadas al embarazo y anticoncepción. 
Se definió intoxicación a la exposición a sustancias posiblemente tóxicas y sus posibles efectos.

En la categoría otros se incluyeron las patologías hematooncológicas, endocrinológicas, oftalmológicas, cardiovasculares y las no clasificables.

El trabajo fue evaluado y aprobado por los comités de ética de las distintas instituciones, teniendo en cuenta la confidencialidad de los adolescentes.

De las historias clínicas se obtuvo la fuente de datos.

Las variables cualitativas se expresaron en frecuencias absolutas y relativas. Las variables cuantitativas se describieron con medidas de tendencia central y de dispersión. En la comparación de proporciones se utilizó el test de chi cuadrado. Se consideró estadísticamente significativo $\mathrm{p}<0,05$. Para el procesamiento de datos se utilizaron los programas Excel y Epi Info 2011.

\section{Resultados}

En el período analizado hubo 17.057 consultas en los servicios de emergencia. Se registraron 1.518 consultas de adolescentes (9\%), correspondiendo al sector público $526(35 \%)$ y al sector privado $992(65 \%)$.

La media de edad fue 13 años 1 mes, mediana 13 años 2 meses, moda 11 años.

Del total de las consultas, $1.041(68,5 \%)$ fueron de adolescentes tempranos (10-14años); 766 (50,5\%) fueron varones.

La distribución de consultas en la semana de cada mes fue la siguiente: enero $300(20 \%)$, abril 447 (30\%), julio 309 (20\%), octubre $462(30 \%)$.

Presentaron comorbilidades 400 (26\%): $34 \%$ patologías respiratorias; $21 \%$ otros; $17 \%$ patologías psiquiátricas; $14 \%$ patologías endócrinas; $10 \%$ patologías neurológicas, y 4\% situaciones de abuso (alcohol o sustancias).

En la tabla 1 se muestran las características de la población.

Analizando los motivos de consulta los más frecuentes fueron: lesiones e injurias 502 (33\%), síntomas respiratorios $216(14 \%)$, otros $205(13,5 \%)$, síntomas digestivos 190 (12,5\%), lesiones en piel 109 (7,0\%).

Se realizaron consultas con especialista en 574 casos (38\%): traumatólogo en 277 (18,2\%); cirujano en 82 $(5,4 \%)$; otorrinolaringólogo en 54 (3,6\%); oftalmólogo en $46(3 \%)$; psiquiatra en $44(3 \%)$; cirujano plástico en 27 ( $1,8 \%)$; ginecólogo en 25 ( $1,6 \%)$; odontólogo en 13 $(0,8 \%)$; en centro toxicológico $7(0,5 \%)$, y otros, 7 $(0,5 \%)$.

Exámenes de laboratorio fueron solicitados en 242 casos (16\%), solicitud de imágenes en 457 (30\%).

Los diagnósticos finales fueron: trauma $505(33 \%)$, patología respiratoria $308(20 \%)$, otros $206(13 \%)$, patología digestiva 147 (10\%), patología de piel $100(6,6 \%)$, patología psicosocial $70(5,0 \%)$, patología urinaria 53 $(3,5 \%)$, patología neurológica $47(3,1 \%)$, patología quirúrgica $28(2,0 \%)$, consultas sobre salud sexual y reproductiva $28(2,0 \%)$, intoxicaciones $8(0,5 \%)$, se reti$\mathrm{ran} / \mathrm{sin}$ datos $18(1,1 \%)$.

La tabla 2 muestra los diagnósticos finales y destino en los sectores público y privado.

La tabla 3 muestra en el sector privado la comparación entre diagnósticos finales y destino, según adolescencia temprana y tardía.

Las tablas 4 y 5 muestran la relación entre edad y diagnósticos finales según estación del año.

Cuando se compara el diagnóstico final según el sexo, si bien el orden de frecuencia es el mismo en los primeros cinco diagnósticos, el número de consultas es estadísticamente significativo, destacándose la mayor consulta por trauma en varones mientras que en las mujeres aumenta en la patología digestiva (tabla 6).

Es de destacar que de las consultas con diagnóstico de patología psicosocial, el 63\% de los pacientes presentaba como comorbilidad la presencia de patología psicosocial.

El destino final de los pacientes fue: domicilio: 1.364 (90\%); hospitalización: 134 (8,8\%); se retiran antes de la asistencia: $14(0,9 \%)$; institucionalizados: 2 $(0,1 \%)$; sin datos: $4(0,2 \%)$.

No falleció ningún adolescente.

\section{Discusión}

Este trabajo constituye la primera descripción de motivos de consulta adolescente en servicios de emergencia en nuestro país.

Las consultas adolescentes correspondieron a 9\%, cifra que coincide con la bibliografía analizada ${ }^{(9,10)}$, lo que refleja que esta población es relativamente "sana", si bien se enfrentan a comportamientos de riesgo y estilos de vida no saludables que pueden determinar lesiones o enfermedades que requieran de la utilización de la urgencia.

El HP-CHPR es el centro de mayor complejidad asistencial para los niños de hasta 15 años de edad de la red de servicios de la Administración de los Servicios de Salud del Estado (ASSE) de todo el país, atendiendo a la población de mayor vulnerabilidad. Esto puede sesgar los motivos de consultas.

No es posible obtener datos concluyentes en relación con la frecuencia de consultas entre adolescencia temprana y tardía en ambos sectores de salud debido a que en el sector público se asiste solo hasta los 15 años. En las consultas del sector privado se incluyeron pacientes de hasta 19 años, lo que permitió comparar adolescencia temprana y tardía, la frecuencia de consultas es similar en las dos etapas. 
Tabla 1. Consultas de adolescentes en servicios de emergencia. Características de la población. Año 2013.

\begin{tabular}{|c|c|c|c|}
\hline Variable & Categorías & $F A(n)$ & $F R(\%)$ \\
\hline \multirow[t]{2}{*}{ Población (n 1.518) } & 10-14 años & 1.041 & $68,5 \%$ \\
\hline & 15-19 años & 477 & $31,5 \%$ \\
\hline \multirow[t]{2}{*}{ Sexo } & Masculino & 766 & $50,5 \%$ \\
\hline & Femenino & 752 & $49,5 \%$ \\
\hline \multirow[t]{2}{*}{ Comorbilidades } & No & 1.118 & $74,0 \%$ \\
\hline & Sí & 400 & $26,0 \%$ \\
\hline \multirow[t]{11}{*}{ Motivo de consulta } & Lesiones e injurias & 502 & $33,0 \%$ \\
\hline & Síntomas respiratorios & 216 & $14,0 \%$ \\
\hline & Síntomas digestivos & 190 & $12,5 \%$ \\
\hline & Lesiones de piel & 109 & $7,0 \%$ \\
\hline & Fiebre & 94 & $6,0 \%$ \\
\hline & Psicosociales & 58 & $4,0 \%$ \\
\hline & Síntomas neurológicos & 58 & $4,0 \%$ \\
\hline & Síntomas urinarios & 45 & $3,0 \%$ \\
\hline & Síntomas de la esfera sexual & 29 & $2,0 \%$ \\
\hline & Ingesta de fármacos & 12 & $1,0 \%$ \\
\hline & Otros & 205 & $13,5 \%$ \\
\hline \multirow[t]{3}{*}{ Consulta con especialistas } & No & 926 & $61,0 \%$ \\
\hline & Sí & 574 & $38,0 \%$ \\
\hline & Se retiran/Sin dato & 18 & $1,0 \%$ \\
\hline \multirow[t]{3}{*}{ Exámenes de laboratorio } & Sí & 242 & $16,0 \%$ \\
\hline & No & 1.258 & $83,0 \%$ \\
\hline & Se retiran/Sin dato & 18 & $1,0 \%$ \\
\hline \multirow[t]{3}{*}{ Imágenes } & Sí & 457 & $30,0 \%$ \\
\hline & No & 1.043 & $69,0 \%$ \\
\hline & Se retiran/Sin dato & 18 & $1,0 \%$ \\
\hline \multirow[t]{8}{*}{ Diagnóstico final } & Trauma & 505 & $33,0 \%$ \\
\hline & Patología respiratoria & 308 & $20,0 \%$ \\
\hline & Patología digestiva & 147 & $10,0 \%$ \\
\hline & Patología de piel & 100 & $6,6 \%$ \\
\hline & Patología psicosocial & 70 & $5,0 \%$ \\
\hline & Intoxicaciones & 8 & $0,5 \%$ \\
\hline & Otros & 362 & $23,8 \%$ \\
\hline & Se retiran/Sin dato & 18 & $1,1 \%$ \\
\hline \multirow[t]{5}{*}{ Destino } & Domicilio & 1.364 & $90 \%$ \\
\hline & Hospitalización & 134 & $8,8 \%$ \\
\hline & Institucionalizados & 2 & $0,1 \%$ \\
\hline & Se retira previo a la asistencia & 14 & $0,9 \%$ \\
\hline & Sin datos & 4 & $0,2 \%$ \\
\hline
\end{tabular}

FA: frecuencia absoluta, FR: frecuencia relativa. 
Tabla 2. Consultas de adolescentes en servicios de emergencia. Diagnóstico final y destino según sector de asistencia público y asistencia médica colectiva. Año 2013.

\begin{tabular}{|c|c|c|c|c|c|c|}
\hline \multirow[t]{3}{*}{ Variable } & \multirow[t]{3}{*}{ Categorías } & \multicolumn{5}{|c|}{ 10-14 años ( $n=1041)$} \\
\hline & & \multicolumn{2}{|c|}{ Público $n=526$} & \multicolumn{2}{|c|}{ Privado $n=515$} & \multirow[b]{2}{*}{$p$} \\
\hline & & $F A(n)$ & $F R(\%)$ & $F A(n)$ & $F R(\%)$ & \\
\hline \multirow[t]{8}{*}{ Diagnóstico final } & Trauma & 203 & $38,6 \%$ & 162 & $31,5 \%$ & $<0,05$ \\
\hline & Patología respiratoria & 91 & $17,3 \%$ & 135 & $26,2 \%$ & $<0,01$ \\
\hline & Patología digestiva & 44 & $8,4 \%$ & 55 & $10,7 \%$ & NS \\
\hline & Patología de piel & 36 & $6,8 \%$ & 28 & $5,4 \%$ & NS \\
\hline & Patología psicosocial & 38 & $7,2 \%$ & 9 & $1,7 \%$ & $<0,01$ \\
\hline & Intoxicaciones & 7 & $1,3 \%$ & 1 & $0,2 \%$ & $<0,05$ \\
\hline & Otros & 100 & $19,0 \%$ & 124 & $24,1 \%$ & NS \\
\hline & Se retiran & 7 & $1,3 \%$ & 1 & $0,2 \%$ & $<0,05$ \\
\hline \multirow[t]{3}{*}{ Destino } & Domicilio & 452 & $85,9 \%$ & 473 & $91,8 \%$ & $<0,01$ \\
\hline & Hospitalización & 67 & $12,7 \%$ & 41 & $8,0 \%$ & $<0,01$ \\
\hline & Se retira previo a asistencia & 7 & $1,3 \%$ & 1 & $0,2 \%$ & $<0,05$ \\
\hline
\end{tabular}

NS: no significativo; $<0,05$ significativo; $<0,01$ muy significativo.

FA: frecuencia absoluta, FR: frecuencia relativa.

Tabla 3. Consultas de adolescentes en servicios de emergencia de los servicios de asistencia médica colectiva. Comparación del diagnóstico al egreso y destino final, según etapa de adolescencia. Año 2013.

\begin{tabular}{|c|c|c|c|c|c|c|}
\hline \multirow[b]{2}{*}{ Variable } & \multirow[b]{2}{*}{ Categorías } & \multicolumn{2}{|c|}{$10-14$ años $n=515$} & \multicolumn{2}{|c|}{$15-19$ años $n=477$} & \multirow[t]{2}{*}{$p$} \\
\hline & & $F A(n)$ & $F R(\%)$ & $F A(n)$ & $F R(\%)$ & \\
\hline \multirow[t]{9}{*}{ Diagnóstico final } & Trauma & 162 & $31,4 \%$ & 140 & $29,3 \%$ & NS \\
\hline & Patología respiratoria & 135 & $26,2 \%$ & 82 & $17,2 \%$ & $<0,01$ \\
\hline & Patología digestiva & 55 & $10,7 \%$ & 48 & $10,1 \%$ & NS \\
\hline & Patología de piel & 28 & $5,4 \%$ & 36 & $7,5 \%$ & NS \\
\hline & Patología psicosocial & 9 & $1,7 \%$ & 23 & $4,8 \%$ & NS \\
\hline & Intoxicaciones & 1 & $0,2 \%$ & 0 & $0,0 \%$ & NS \\
\hline & Otros & 124 & $24,0 \%$ & 138 & $28,9 \%$ & NS \\
\hline & Se retiran & 1 & $0,2 \%$ & 6 & $1,3 \%$ & NS \\
\hline & Sin datos & 0 & $0,0 \%$ & 4 & $0,8 \%$ & NS \\
\hline \multirow[t]{5}{*}{ Destino } & Domicilio & 473 & $91,8 \%$ & 441 & $92,4 \%$ & NS \\
\hline & Hospitalización & 41 & $7,9 \%$ & 26 & $5,5 \%$ & NS \\
\hline & Institucionalizados & 0 & $0,0 \%$ & 2 & $0,4 \%$ & NS \\
\hline & Se retira previo a la asistencia & 1 & $0,2 \%$ & 6 & $1,3 \%$ & NS \\
\hline & Sin datos & 0 & $0,0 \%$ & 2 & $0,4 \%$ & NS \\
\hline
\end{tabular}

NS: no significativo; $<0,05$ significativo; $<0,01$ muy significativo; FA: frecuencia absoluta; FR: frecuencia relativa. 
Tabla 4. Consultas de adolescentes en servicios de emergencia. Diagnóstico final según meses del año (enero/abril), comparación grupo etario. Año 2013.

\begin{tabular}{|c|c|c|c|c|c|c|}
\hline \multirow{2}{*}{$\begin{array}{l}\text { Mes del año } \\
(n=1.518)\end{array}$} & \multirow[t]{2}{*}{ Diagnóstico final } & \multicolumn{2}{|c|}{10 a 14 años } & \multicolumn{2}{|c|}{15 a 19 años } & \multirow[t]{2}{*}{$P$} \\
\hline & & $F A(n)$ & $F R(\%)$ & $F A(n)$ & $F R(\%)$ & \\
\hline \multirow[t]{8}{*}{ Enero } & Patología respiratoria & 69 & 23,00 & 10 & 4,00 & $<0,05$ \\
\hline & Trauma & 64 & 21,00 & 22 & 7,00 & NS \\
\hline & Otros & 47 & 15,67 & 16 & 3,00 & NS \\
\hline & Patologías digestivas & 20 & 7,00 & 6 & 2,00 & NS \\
\hline & Patología de piel & 21 & 6,50 & 9 & 3,00 & NS \\
\hline & Patología psicosocial & 9 & 3,00 & 4 & 2,00 & NS \\
\hline & Intoxicaciones & 1 & 0,25 & 0 & 0,00 & NS \\
\hline & Sin datos & 1 & 0,25 & 1 & 0,25 & NS \\
\hline$(n=300)$ & $\mathrm{n}$ total & 232 & 77,00 & 68 & 23,00 & \\
\hline \multirow[t]{8}{*}{ Abril } & Trauma & 115 & 26,00 & 54 & 12,00 & NS \\
\hline & Otros & 78 & 17,50 & 41 & 9,00 & $<0,05$ \\
\hline & Patología respiratoria & 47 & 10,50 & 21 & 5,00 & NS \\
\hline & Patologías digestivas & 24 & 4,50 & 19 & 4,00 & NS \\
\hline & Patología psicosocial & 13 & 3,25 & 9 & 2,00 & NS \\
\hline & Patología de piel & 13 & 3,00 & 8 & 2,00 & NS \\
\hline & Intoxicaciones & 2 & 0,50 & 0 & 0,00 & NS \\
\hline & Sin dato & 1 & 0,25 & 2 & 0,50 & NS \\
\hline$(n=447)$ & $\mathrm{n}$ total & 293 & 65,50 & 154 & 34,50 & \\
\hline
\end{tabular}

NS: no significativo; $<0,05$ significativo; $<0,01$ muy significativo; FA: frecuencia absoluta, FR: frecuencia relativa.

Las consultas durante los cuatro meses correspondieron a $20 \%-30 \%$, no encontrando diferencia significativa por estación. Esto evidencia una consulta constante de este grupo etario durante todo el año, llamando la atención en enero la patología respiratoria, que ocupa el primer lugar siendo las principales causas otitis y faringitis, mientras que el trauma predominó en los meses de abril y octubre.

En esta serie no hay predominancia en cuanto a sexo, a diferencia de otros estudios en donde predomina el sexo femenino ${ }^{(11,12)}$

Se evidenció un alto porcentaje de adolescentes con comorbilidades, lo que va en relación con el aumento en el diagnóstico y prevalencia de las enfermedades crónicas en este grupo etario. Esto ha sido constatado por la ENSA y tiene un impacto claro en la calidad de vida de los adolescentes.
En la bibliografía revisada el presentar una enfermedad crónica, sobre todo de la esfera psiquiátrica y abuso de sustancias, es un factor de riesgo para suicidio e intento de autoeliminación (IAE) ${ }^{(2)}$. En el presente análisis las patologías respiratoria y psiquiátrica fueron las principales comorbilidades encontradas.

Se constató un escaso registro en relación con los diagnósticos nutricionales, lo que puede subestimar la prevalencia de malnutrición en esta población. El exceso de peso es el principal problema nutricional en esta etapa de la vida. Los datos obtenidos de la ENSA reflejan $26,6 \%$ de sobrepeso y $7 \%$ de obesidad. De ahí la importancia de contar con este dato, sabiendo que es la enfermedad crónica no transmisible más frecuente en la adolescencia, determinando impacto en la calidad de vida y múltiples consultas en los servicios de salud debido a depresión, IAE y patología cardiovascular. 
Tabla 5. Consultas de adolescentes en servicios de emergencia. Diagnóstico final según meses del año (julio/octubre), comparación según grupo etario. Año 2013.

\begin{tabular}{|c|c|c|c|c|c|c|}
\hline \multirow{2}{*}{$\begin{array}{l}\text { Mes del año } \\
(n=1.518)\end{array}$} & \multirow[t]{2}{*}{ Diagnóstico final } & \multicolumn{2}{|c|}{10 a 14 años } & \multicolumn{2}{|c|}{15 a 19 años } & \multirow[t]{2}{*}{$P$} \\
\hline & & $F A(n)$ & $F R(\%)$ & $F A(n)$ & $F R(\%)$ & \\
\hline \multirow[t]{8}{*}{ Julio } & Patología respiratoria & 55 & 18,00 & 28 & 9,00 & NS \\
\hline & Trauma & 53 & 17,00 & 27 & 8,50 & NS \\
\hline & Otros & 39 & 13,00 & 39 & 13,00 & $<0,05$ \\
\hline & Patología psicosocial & 14 & 4,50 & 4 & 1,00 & NS \\
\hline & Patologías digestivas & 13 & 4,00 & 12 & 3,75 & NS \\
\hline & Patología de piel & 10 & 3,00 & 9 & 2,75 & NS \\
\hline & Intoxicaciones & 3 & 1,00 & 0 & 0,00 & NS \\
\hline & Sin datos & 1 & 0,50 & 2 & 1,00 & NS \\
\hline $\mathrm{n}=(309)$ & $\mathrm{n}$ total & 188 & 61,00 & 121 & 39,00 & \\
\hline \multirow[t]{8}{*}{ Octubre } & Trauma & 133 & 29,00 & 38 & 8,00 & $<0,05$ \\
\hline & Otros & 64 & 14,00 & 46 & 10,00 & $<0,01$ \\
\hline & Patología respiratoria & 56 & 12,00 & 22 & 4,75 & NS \\
\hline & Patologías digestivas & 42 & 9,00 & 11 & 2,00 & NS \\
\hline & Patología de piel & 19 & 3,75 & 11 & 2,00 & NS \\
\hline & Patología psicosocial & 11 & 2,00 & 6 & 1,25 & NS \\
\hline & Intoxicaciones & 2 & 0,50 & 0 & 0,00 & NS \\
\hline & Sin datos & 5 & 0,75 & 4 & 1,00 & NS \\
\hline$(n=462)$ & $\mathrm{n}$ total & 327 & 71,00 & 134 & 29,00 & \\
\hline
\end{tabular}

NS: no significativo; $<0,05$ significativo; $<0,01$ muy significativo; FA: frecuencia absoluta; FR: frecuencia relativa

Los traumatismos ocupan el primer lugar tanto en los motivos de consulta como en el diagnóstico final en esta serie $^{(11,13,14)}$, reflejando la importancia de enfatizar en la prevención de los mismos, para evitar la principal causa de muerte en nuestro país ${ }^{(15)}$.

Es importante destacar que existió una diferencia muy significativa cuando comparamos el sexo en relación con el diagnóstico final en trauma, predominando este en los varones. Mientras que la patología digestiva predomina en el sexo femenino, lo cual puede deberse a que las algias abdominales pueden corresponder a etiología funcional o genitourinaria. En cuanto a la patología psicosocial, no hubo diferencia significativa en relación con el sexo, lo cual refleja la importancia de pesquisar factores de riesgo en ambos sexos. Resaltamos que predominan los intentos de autoeliminación en el sexo femenino, mientras que el suicidio consumado es mayor en varones según datos internacionales ${ }^{(5)}$.

Se constató un alto porcentaje de utilización de especialistas, predominando la interconsulta con traumatólogo, acorde al principal motivo de consulta y diagnóstico final. Al igual que la utilización de la imagenología.

En relación con la utilización de exámenes complementarios es importante destacar que el $46 \%$ de las consultas requirió valoración con exámenes de laboratorio o imagen, dato que es superior a lo descrito en la bibliografía analizada $^{(11)}$. Esto demuestra la necesidad de mejorar el contexto en el cual se realiza la historia clínica del adolescente como parte de la entrevista, creando un ambiente adecuado que permita pesquisar procesos que puedan pasar inadvertidos y eviten la necesidad de exámenes complementarios. También interesa resaltar la 
Tabla 6. Consultas de adolescentes en servicios de emergencia. Diagnóstico final según sexo. Año 2013.

\begin{tabular}{lccccc}
\hline Diagnóstico final & \multicolumn{2}{c}{ Varones $(n=766)$} & \multicolumn{2}{c}{ Mujeres $(n=752)$} & $P$ \\
\cline { 2 - 5 } & $F A(n)$ & $F R(\%)$ & $F A(n)$ & FR (\%) & \\
\hline Trauma & 335 & 43,73 & 171 & 22,73 & $<0,01$ \\
Respiratorio & 153 & 19,97 & 155 & 20,61 & NS \\
Otros & 131 & 11,225 & 231 & 15,02 & $<0,01$ \\
Digestivo & 58 & 57,7 & 89 & 11,83 & $<0,01$ \\
Piel & 50 & 6,52 & 50 & 6,64 & NS \\
Psicosocial & 31 & 4,04 & 39 & 5,48 & NS \\
Sin datos & 5 & 0,65 & 12 & 1,59 & NS \\
Intoxicaciones & 3 & 0,39 & 5 & 0,66 & NS \\
\hline
\end{tabular}

NS: no significativo; $<0,05$ significativo; $<0,01$ muy significativo; FA: frecuencia absoluta; FR: frecuencia relativa.

complejidad del proceso diagnóstico en este rango etario.

Destacamos que al comparar los diagnósticos finales entre los sectores público y privado hay diferencia significativa en los diagnósticos de trauma, patología psicosocial e intoxicaciones, predominando en el sector público. Esto puede deberse a la mayor vulnerabilidad social de este sector.

Hay un porcentaje importante de enfermedades inespecíficas, datos que coinciden con la bibliografía analizada $^{(11)}$. Esto refleja la complejidad de esta etapa de la vida, con múltiples cambios físicos y psicológicos, manifestaciones psicosomáticas, que motivan consultas sin una causa orgánica clara.

Con respecto al destino final destacamos que si bien la principal causa de consulta fue traumática, la mayoría fueron traumas leves, dado que se derivaron a domicilio en la mayor parte de los casos y no hubo muertes. Como defecto del análisis dentro de las hospitalizaciones, no se discriminaron los ingresos a cuidados intensivos. Respecto a la admisión hospitalaria, según la bibliografía analizada, los adolescentes tienen un porcentaje mayor de admisión que otros grupos etarios ${ }^{(11)}$, siendo muy significativa la diferencia de admisión hospitalaria entre los sectores, predominando las admisiones en el sector público en nuestra serie.

Una de las limitantes del trabajo fue el deficiente registro en las historias clínicas, en especial en la adolescencia tardía. Destacamos que en nuestro país los adolescentes tardíos son atendidos en servicios de emergencia de adultos, habitualmente por personal no capacitado en adolescentes.
Otra limitante importante fue no analizar de qué manera llegaban los adolescentes a la emergencia, debido a que el trabajo fue retrospectivo y este dato no es obligatorio en los registros. Es un dato importante a analizar a futuro, si concurren solos o acompañados, o son traídos por servicios de emergencia o agentes policiales.

Es importante constatar en los registros si se respetan los derechos de los adolescentes, pidiendo consentimiento para extracción de estudios y maniobras.

\section{Conclusiones}

Si bien la consulta de adolescentes es de baja prevalencia, la misma genera un impacto en los servicios de urgencia por la elevada utilización de recursos, con un porcentaje importante de síntomas inespecíficos. Creemos que el adolescente requiere que los servicios de urgencia estén preparados para su asistencia, favoreciendo un abordaje integral con espacios y personal capacitado.

\section{Summary}

The WHO defines an adolescent as any person between ages 10 and 19 (early adolescence being between 10 and 14 years old, late adolescence being between 15 and 19 years). The prevalence of diseases in adolescents is low, although they use the emergency services and thus require a comprehensive and multidisciplinary approach. No data has been published on the reasons for the consultation of adolescents in Uruguay.

Objective: the aim of this study was to determine the frequency and characteristics of adolescent consulta- 
tions at the emergency services in the public and private subsector of the national integrated health system.

Method: multicenter, retrospective study of adolescents between ages 10 and 14 years old who consulted at the Pereira Rossell Hospital HP-CHPR (public sector) and between 10 and 19 years old in $\mathrm{AE}, \mathrm{CAMOC}, \mathrm{CG}$ (private sector). The study included consultations from 7th to 13th January, April, July and October 2013, and the following data were recorded: age, sex, chief complaint, comorbidities, specialist consultations, laboratory tests, diagnosis and destination upon discharge. Statistical analysis was performed, being $p<0.05$ statistically important.

Results: $\mathrm{n}=1518$, representing $9 \%$ of all consultations. Age: mean 13 years 1 month, male: 50.5\%; comorbidities: $26 \%$. Public sector 35\%; private sector: $65 \%$. Distribution of consultations per month: January $20 \%$, April $30 \%$, May $20 \%$, October $30 \%$. The most frequent reasons for consultation were lesions and injuries $33 \%$, respiratory symptoms $14 \%$, other $13 \%, 12.5 \%$ gastrointestinal symptoms, skin lesions 7\%. Psycho-social problems represented $7.2 \%$ in the public sector and $3.2 \%$ in the public sector. Consultation with a specialist $38 \%$; laboratory: $16 \%$; imaging techniques $30 \%$. Main diagnoses upon discharge: $33 \%$ trauma, $20 \%$ respiratory disease, $13 \%$ other, $10 \%$ gastrointestinal pathology, $6.6 \%$ pathology of skin $6,6 \%$. Final destination: $90 \%$ home, $13 \%$ hospital in the public sector, $8 \%$ hospital in the private sector, $0.9 \%$ leave the emergency service before assistance, $0.1 \%$ institutionalized.

Conclusions: while adolescent consultation has a low prevalence, it has a significant impact on emergency services due to its use of resources. Differences are observed between the public and private sector.

\section{Resumo}

A OMS define adolescência como a etapa compreendida entre os 10 e 19 anos (precoce: 10 e 14 anos; tardia: 15 a 19 anos). Embora a prevalência de doenças na adolescência seja baixa os adolescentes utilizam serviços de emergência, o que requer uma abordagem integral e multidisciplinar. Não existem dados publicados sobre os motivos de consultas dos adolescentes no Uruguai.

Objetivo: o objetivo deste estudo é conhecer a frequência e as características da consulta de adolescentes nos serviços de emergência do subsetor público e privado do sistema nacional integrado de saúde.

Metodología: estudo multicêntrico, retrospectivo de adolescentes entre 10-14 anos que consultaram no HP-CHPR (Hospital Pediátrico Centro Hospitalario Pereira Rossell da Administración de Salud del Estado (ASSE): subsetor público) e entre 10-19 anos na AE (Asociación Española Primera de Socorros Mutuos),
CAMOC (Centro de Asistencia Médica del Oeste de Colonia), CG (Casa de Galicia) (Instituições de Assistência Médica Coletiva: IAMC). Foram incluídas as consultas dos dias 7 a 13 dos meses janeiro, abril, julho e outubro de 2013. Foram registrados: idade, sexo, motivo de consulta, comorbidades, consultas com especialistas, exames de laboratório, diagnóstico e destino na alta. Foi realizada uma análise estadística considerando estatisticamente significativo $\mathrm{p}<0.05$.

Resultados: $\mathrm{n}=1518,9 \%$ do total de consultas. Idade: média 13 anos e 1 mês, 50,5\% eram do sexo masculino e $26 \%$ apresentavam comorbidades. $35 \%$ foram atendidos no subsetor público e $65 \%$ no subsetor privado (IAMC). A distribuição mensal de consultas foi: janeiro $20 \%$, abril $30 \%$, julho $20 \%$, outubro $30 \%$. Os motivos de consulta mais frequentes foram lesões $33 \%$, sintomas respiratórios $14 \%$, outros $13 \%$, sintomas digestivos $12,5 \%$, lesões na pele $7 \%$. Os problemas psicossociais ccorrespondiam a $7,2 \%$ no setor público, e a 3,2\% no privado. Consulta com especialista 38\%. Laboratorio: $16 \%$. Imagens $30 \%$. Diagnósticos principais na alta: trauma $33 \%$, patologia respiratória $20 \%$, outros $13 \%$, patologia digestiva $10 \%$, patologia de pele $6,6 \%$. Destino final: domicilio $90 \%$, hospitalização: $13 \%$ no subsetor público e $8 \%$ nas IAMC. $0,9 \%$ se retira antes da assistência, $0,1 \%$ se institucionaliza.

Conclusões: embora a consulta de adolescentes tenha baixa prevalência, esta gera um impacto nos serviços de urgência pela elevada utilização de recursos. Foram observadas diferenças entre o setor público e privado.

\section{Bibliografía}

1. Cromer B, Bockting W, Remafedi G, Burstein GR, Stager MM, Elfenbein DS, et al. Medicina de la adolescencia, parte XIII. En: Kliegman RM, Stanton BF, Schor NF, St Geme II JW, Behrman RE, eds. Nelson tratado de pediatría: volumen 1. 19 ed. Ámsterdam: Elsevier; 2013:680-749.

2. Organización Panamericana de la Salud. Estrategia y plan de acción regional sobre los adolescentes y jóvenes 2010-2018. Washington, DC: OPS, 2010.

3. Uruguay. Ministerio de Salud Pública. Programa Nacional de Salud Adolescente. Guías para el abordaje integral de la salud de adolescentes en el primer nivel de atención. Montevideo: MSP, 2009. Dispopnible en: http://www.msp. gub.uy/sites/default/files/archivos_adjuntos/Guia_abordaje_salud_adolescente_08_10\%5B $1 \% 5$ D.pdf. [Consulta: 30 agosto 2014].

4. Hidalgo Vicario MI, Redondo Romero AM, Castellano Barca G. Medicina de la adolescencia: atención integral. Madrid: Ergon, 2012.

5. Vázquez M, García L, Giachetto G, Martínez A, Viola L. Intentos de autoeliminación en niños y adolescentes. Opción Médica 2012; 22(6):12-5. 
6. Organización Mundial de la Salud. Salud de los adolescentes. Ginebra: OMS, 2014. Disponible en: http://www. who.int/topics/adolescent_health/es/ [consulta: 30 agosto 2014].

7. Garcia L, De Olivera N, Grunbaum S, Giachetto G. Desafíos en la atención integral de los adolescentes. Opción Médica $2013 ; 28(8): 30-3$.

8. Melzer-Lange M, Lye PS. Adolescent health care in a pediatric emergency department. Ann Emerg Med 1996; 27(5):633-7.

9. Bianco M, Correa C. Adolescentes: motivos de consulta. Salta: Universidad Nacional de Salta, 2003. Disponible en: $\mathrm{http} / / \mathrm{html}$.rincondelvago.com/motivos-de-consulta-de-adolescentes.html. [Consulta: 10 setiembre 2014].

10. Míguez H, Fernández AR, Gordillo ME, Casella E, Quinteros R, Mansilla JC. Consulta de emergencia en niños y adolescentes por abuso de sustancias psicoactivas. Rev. Salud Pública (Córdoba) 2012; 16(1):8-17.
11. Ruiz España A, García García JJ, Luaces Cubells C, Garrido Romero R, Pou Fernández J. Enfermedades del adolescente en el servicio de urgencias. An Pediatr 2001; 54(3):238-42.

12. Peñas Pascual N, Sainz Camuñas MP, Puche López N. Urgencias hospitalarias en los adolescentes. Emergencias 1990; 2(3):121-7.

13. Ziv A, Boulet JR, Slap GB. Emergency department utilization by adolescents in the United States. Pediatrics 1998; 101(6):987-94.

14. Moiset Homs F, Bonamussa Ventura A, Plaja Román P. Urgencias pediátricas en un hospital comarcal. Emergencias 1994; 6(4):167-71.

15. Uruguay. Ministerio de Salud Pública. Epidemiología. Vigilancia en salud. Tasa de mortalidad de adolescentes y jóvenes. Montevideo: MSP, 2011. Disponible en: www.msp.gub.uy. [Consulta: 5 setiembre 2014]. 